\title{
Visual search and contextual cueing: differential effects in 10-year-old children and adults
}

\author{
Jane W. Couperus • Ruskin H. Hunt • \\ Charles A. Nelson • Kathleen M. Thomas
}

Published online: 11 November 2010

(C) Psychonomic Society, Inc. 2010

\begin{abstract}
The development of contextual cueing specifically in relation to attention was examined in two experiments. Adult and 10-year-old participants completed a context cueing visual search task (Jiang \& Chun, The Quarterly Journal of Experimental Psychology, 54A(4), 1105-1124, 2001) containing stimuli presented in an attended (e.g., red) and unattended (e.g., green) color. When the spatial configuration of stimuli in the attended and unattended color was invariant and consistently paired with the target location, adult reaction times improved, demonstrating learning. Learning also occurred if only the attended stimuli's configuration remained fixed. In contrast, while 10 year olds, like adults, showed incrementally slower reaction times as the number of attended stimuli increased, they did not show learning in the standard paradigm. However, they did show learning when the ratio of attended to unattended stimuli was high, irrespective of the total number of attended stimuli. Findings suggest children show efficient attentional guidance by color in visual search but differences in contextual cueing.
\end{abstract}

Keywords Selective attention - Contextual cueing Development · Visual search · Implicit learning

J. W. Couperus $(\bowtie)$

Adele Simmons Hall, Cognitive Sciences, Hampshire College, Amherst, MA 01002, USA

e-mail: jcouperus@hampshire.edu

R. H. Hunt $\cdot$ K. M. Thomas

Institute of Child Development, University of Minnesota, Minneapolis, MN, USA

R. H. Hunt $\cdot$ K. M. Thomas

Center for Neurobehavioral Development,

University of Minnesota,

Minneapolis, MN, USA

C. A. Nelson

Children's Hospital Boston/Harvard Medical School,

Boston, MA, USA
Selective attention can be defined as the process by which we distribute attentional resources, select, and prioritize information for further processing. Across childhood, our ability to attend selectively to different aspects of the environment improves (Columbo, 2001; Plude, Enns, \& Brodeur, 1994; Ridderinkhoff \& van der Stelt, 2000; Rueda et al., 2004). However, although multiple studies have shown development within selective attention, very few studies have examined how attention development impacts other processes such as implicit learning (e.g., Rosas et al., 2010). As research suggests that attention may influence implicit learning in adults (e.g., Gilbert, 2009; Jiang \& Chun, 2001; Nissen \& Bullemer, 1987), it is likely that the development of selective attention influences this relationship.

The interaction of selective attention and implicit learning is complex. While evidence that attention is important for implicit learning can be seen in a variety of studies (e.g., Jiang \& Chun, 2001; Nissen \& Bullemer, 1987), questions remain regarding this relationship. For example, studies of divided attention (e.g., Nissen \& Bullemer, 1987) typically show decrements in implicit learning when a secondary task is required. However, it may be the expression of learning rather than the learning itself that is impaired (Cohen, Ivry, Keele, 1990; Frensch, Lin, \& Buchner, 1998; Frensch, Wenke, \& Ruenger, 1999; Shanks \& Channon, 2002; Stadler, 1995). Additionally, studies that utilize tasks that do not require divided attention suggest that under certain conditions nonselected material is processed despite efficient selection (e.g., Jiang \& Chun, 2001) or, as in divided attention tasks, may be present but not expressed (e.g., Jiang \& Leung, 2005). However, despite the complexity of the interaction, it is clear that selective attention is important for the expression of implicit learning. Thus, to begin to explore the effect of development on this relation, this study examines the influence of selective attention development 
on the subsequent expression of implicit learning (as distinguished from the learning itself).

Research suggests that selective attention in children undergoes development in early childhood (e.g., for a review see Ridderinkhof \& van der Stelt, 2000). In particular, children's ability to select out the relevant information (Enns \& Girgus, 1985) and ignore irrelevant information (i.e., filtering) (Enns \& Akhtar, 1989; Rueda et al., 2004; McDermott, Pérez-Edgar, \& Fox, 2007) changes across development. However, visual search tasks that involve the guidance of attention by relevant search criteria while ignoring irrelevant features may approach adult levels of performance at approximately 10 years of age, with little additional improvement in subsequent years (e.g., Hommel, Li, \& Li, 2004; Klenberg, Korkman, \& Lahti-Nuuttila, 2001; Taylor \& Khan, 2000). Similarly, research suggests that implicit learning is relatively if not fully mature early in childhood (e.g., Meulemans, Van der Linden, \& Perruchet, 1998; Thomas \& Nelson, 2001).

For example, a recent study by Dixon, Zelazo, and De Rosa (2010) used a contextual cueing paradigm modified for children ( 5 to 9 years of age) to explore memory-guided attention that relies on implicit learning. In the standard contextual cueing paradigm (e.g., Chun \& Jiang, 1998) participants are asked to locate a target letter among a set of distractors and determine the direction that the letter is facing. Unbeknownst to the participants, in some of the displays the target and distractor stimuli are placed at random ("new" condition), and in others the placement of distractors and target remain fixed throughout the experiment ("old" condition). Across the course of the experiment participant reaction times decrease for both conditions, but reaction times are significantly faster in the "old" compared to the "new" condition, demonstrating learning of the fixed or repeating elements. In the Dixon et al. (2010) study, this general paradigm was utilized with age-appropriate stimuli containing additional color and shape contextual features (i.e., pictures of fish) and a simplified spatial context (i.e., all possible spatial locations contained stimuli rather than a subset of locations) to explore contextual cueing in school age children. While this study was able to show learning, an earlier study by Vaidja, Huger, Howard, and Howard (2007) using the standard stimuli has suggested that implicit learning in the contextual cueing task may not be fully developed in school age children. While there are several differences between these studies that may account for differences in learning, one of several explanations put forth by Vaidja et al. for the lack of learning in their task was the potential contribution of immature attentional processes. One way to explore this possibility is to modify the task to include an attentional manipulation as was done by Jiang and Chun (2001). To examine the effects of modulations of attention in the contextual cueing paradigm,
Jiang and Chun (2001) presented two sets of distractors, one set of distractors that were the same color as the target (attended) and a second set (unattended) that were another color (e.g., red and green). This variation allowed for four combinations of attention (unattended/attended) and distractor placement (new/old) to be examined. Findings showed that when participants were attending to the color in which distractors appeared at old locations, learning occurred (as demonstrated through faster reaction times). However, when distractors appeared in old locations but were in the unattended color, learning did not occur. Interestingly, findings from this study suggest that although participants were "seeing" both attended and unattended distractors, they were selectively attending to only the distractors of the target color, and thus only showed learning when the old location information occurred in the attended color. Participants did not learn the old location information if it was in the unattended color.

Similar to typical visual search tasks, attention, specifically attentional guidance by color, in this paradigm can be measured by examining changes in reaction times as a function of the total number of attended stimuli. With efficient attentional guidance by color, reaction times should slow as the number of attended stimuli increases (e.g., Treisman \& Gelade, 1980). For example, in a display of 16 stimuli, 8 attended (green) and 8 unattended (red), efficient guidance by color is defined as searching serially through 8 attended stimuli. Moreover, the reaction time to finding the target should increase if the number of attended items is increased to 12 (green) while the number of unattended stimuli is decreased to 4 (red) despite the number of total stimuli remaining constant. While attentional guidance by color is likely not the only factor in the expression of contextual cueing (e.g., Kunar, Flusberg, Horowitz, \& Wolfe, 2007), immaturity in attentional guidance by color is likely to influence the expression of contextual cueing. Contextual cueing can be measured in this task by examining learning in the two conditions in which half of the distractors remain in old locations across trials. Each of these conditions contains the same number of stimuli appearing in old locations, but they differ in relation to attention paid to stimuli appearing at old locations. If efficient contextual cueing is present, learning will occur when stimuli in the attended color remain in old locations across trials and the stimuli in the unattended color are placed at random. Moreover, no learning will be seen if stimuli in the unattended color remain in old locations across trials and those in the attended color are placed at random. However, if contextual cueing is not efficient, either no learning will occur, or learning will occur only when both the attended and unattended distractors remain in old locations across trials. 
The present study explores attentional guidance by color and contextual cueing in children through two experiments. The first experiment explores these processes in adults and 10 year olds using the standard contextual cueing paradigm while varying the number of attended items to determine if attentional guidance by color and contextual cueing are efficient by the age of 10 within this task. Second, as recent theories have suggested that perceptual load (i.e., increases in the amount of relevant attended information) may aid in selective attention (Lavie, 1995), contributions of perceptual load on selective attention were examined in an additional experiment by increasing the overall number of attended items while holding the ratio of attended to unattended items constant.

\section{Experiment 1}

The goal of Experiment 1 was to explore the development of attention, specifically attentional guidance by color, and its effects on implicit learning within the contextual cueing paradigm. Research has shown implicit learning in a contextual cueing task with children 5 to 9 years of age when attention is not manipulated (Dixon et al., 2010). However, research has also suggested that learning in the contextual cueing paradigm is not reliable and may not be fully mature, particularly in younger children (Vaidya et al., 2007). Thus, to provide the best opportunity to observe learning in children, only 10 year olds were included in this study.

As has been done in previous studies with adults (Jiang \& Chun, 2001), the current experiment manipulates selective attention in the contextual cueing task, with one modification. The ratio of attended to unattended stimuli was varied across participants with one third asked to search through 4 of 16 stimuli, another third 8 of 16 stimuli, and a final third 12 of 16 stimuli. It is hypothesized that if attentional guidance by color is sufficiently developed, reaction times will increase as the number of attended items increases in both adults and children. In contrast, if attentional guidance by color is not fully developed in children, reaction times will be based on the total number of stimuli presented (both attended and unattended). Additionally, it is hypothesized that if implicit learning in the form of contextual cueing is sufficiently developed for this task, children, like adults, will show faster reaction times (i.e., learning) when (1) the target as well as both attended and unattended distractor stimuli appear at old locations, and (2) when only attended distractor stimuli appear at old locations (as was found in adults, Jiang \& Chun, 2001). In contrast, if implicit learning in the form of contextual cueing is not fully developed, learning (1) may not occur at all, or (2) may only occur when displays contain both attended and unattended stimuli that remain at old locations across trials, or (3) may occur only when the number of attended stimuli that remain in old locations across trials is high and the number of unattended distractors placed at random is low.

To explore these various possibilities, analyses will test these a priori hypotheses by directly comparing the condition when both attended and unattended distractors appear at random to each of the other conditions (i.e., when both attended and unattended distractors appear at old locations, when only attended distractors appear at old locations, and when only unattended distractors appear at old locations).

\section{Method}

\section{Participants}

The participants for this experiment were 64 10-year-old children and 63 college students from the Minneapolis area. Seven participants were excluded due to physical, psychological, neurological, and learning disorders (4 10-year-old children and 3 adults), leaving 60 child participants (31 female, 29 male, 62 Caucasian, 1 Black/Caucasian, 1 Asian/ Caucasian, mean age $=10$ years 6 months, $\mathrm{SD}=1.50$ months) and 60 adult participants (19 male, 49 female, mean age = 20 years, 10 months, $\mathrm{SD}=35$ months) for data analyses.

The number of participants chosen was based on previous studies that suggest that learning is robust and significant findings can be found with relatively small sample sizes (e.g., 16-17 participants in Jiang \& Chun, 2001). All participants were screened for color blindness and learning disorders (based on self or parent report). Additionally, all adult participants, as well as parents of children, gave written consent, and all 10-year-old children gave written assent, in accordance with the University of Minnesota Institutional Review Board.

\section{Stimuli and design}

Contextual cueing paradigm On each trial a fixation target (a .5-cm white cross) was presented for $500 \mathrm{~ms}$. Following fixation, a search display was presented until the participant responded (see Fig. 1). Stimuli in the display consisted of rotated "L" and "T" shapes that have been used previously with both adults and children (Jiang \& Chun, 2001; Vaidya et al., 2007). For each display participants were asked to find the single rotated " $\mathrm{T}$ " stimulus (the target) and indicate the direction it faced. Responses were recorded using a mouse with both a left and right button. Participants were asked to hold the mouse with two hands, using their thumbs to respond. If the long end of the " $\mathrm{T}$ " was facing right they 
a) $75: 25$

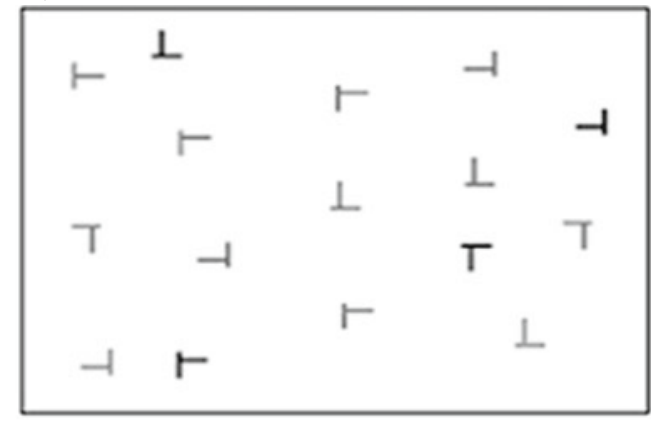

b) $50: 50$

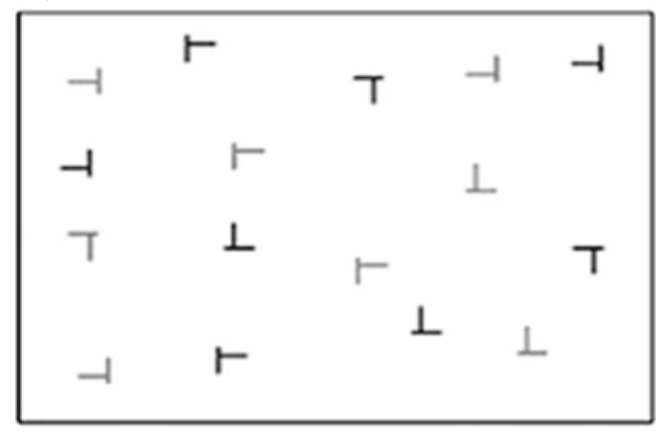

c) $25: 75$

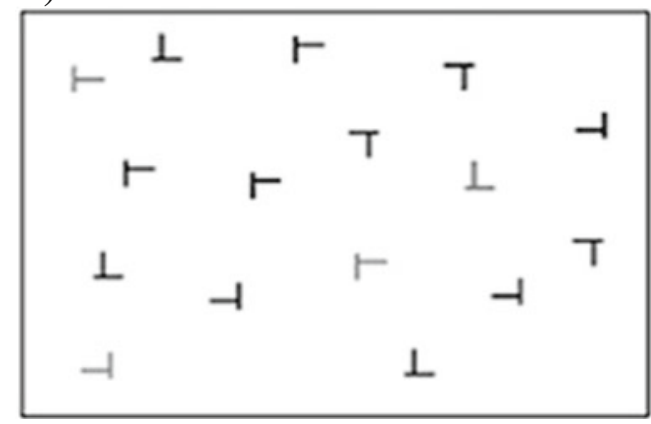

Fig. 1 Example displays for Experiment 1. (a) Example of the 25:75 task. (b) Example of the 50:50 task. (c) Example of the 75:25 task

were asked to press the right button, and if it was facing left, they were asked to press the left button. Feedback was provided for $500 \mathrm{~ms}$ after each response (a high or low tone associated with correct and incorrect responses, respectively), followed by an inter-trial interval ranging from 50 to $100 \mathrm{~ms}$. Each experimental session consisted of 6 epochs of 5 blocks, and each block contained 12 trials, for a total of 360 trials. Participants received a short break (no more than $5 \mathrm{~min}$ ) after each epoch and a longer break (approximately 5 to $10 \mathrm{~min}$ ) after the third epoch.

Line segments of the L's had a small offset $(.25 \mathrm{~cm})$ to increase the difficulty of the search task (see Fig. 1). This was done to ensure that participants (both adults and children) would have to perform a serial search (i.e., could not rely on "pop-out" effects). The stimuli were approxi- mately $1 \mathrm{~cm} \times 1 \mathrm{~cm}$ in size and subtended a visual angle of approximately $1-2$ degrees as participants sat $40 \mathrm{~cm}-60 \mathrm{~cm}$ from the screen. Each screen contained one target (rotated "T") and two sets of distractors (rotated "L"s). L's were rotated 90 degrees (such that they faced either left or right) as well as inverted (to create up and down orientations). Ts were rotated 90 degrees to appear in either left or right orientations (see Fig. 1). One set of distractors appeared in green and the other set appeared in red. One set of distractors contained stimuli in the same color as the target. This set was termed the "attended" set. The other set of distractors was in the opposite color and was termed the "unattended" set. For each participant the color of the attended and unattended sets was fixed throughout the course of the experiment. Additionally, the colors were counterbalanced such that half of the participants were given green as the attended color and half were given red. The screen was divided into a $12 \times 8$ matrix, and the stimuli were arranged in the matrix according to the following parameters: (1) the target stimuli were placed so that there was equal representation of the targets in the four quadrants of the screen across each epoch of trials; (2) the targets were equated for eccentricity (i.e., the target locations were balanced across conditions such that the average distance from the center of the screen was equal for targets across conditions); (3) the distribution of remaining distractors was balanced such that equal numbers of green and red distractors appeared in each quadrant; (4) the direction of the target varied from trial to trial such that it faced left 50\% of the time and right $50 \%$ of the time; (5) the left-right orientation of all distractors varied randomly from trial to trial, with the constraint that $50 \%$ of distractors faced left and $50 \%$ faced right on any given trial. The background was black.

Each block contained four conditions (three trials of each condition in each block). There were 12 target locations in each block ( 3 for each condition) that were fixed across the experiment. Thus, each target location appeared 30 times. The four conditions were created by varying two factors: attention (attended or unattended) and distractor placement (old or new). The four conditions were labeled both-old, attended-old, unattended-old, and both-new. In the both-old condition, both the attended and unattended sets of distractors were placed in the same spatial configuration (old) in relation to the target stimulus for each trial. In the attended-old condition, the attended set (containing the target) contained distractors placed in the same spatial configuration (old) in relation to a given target stimulus for each trial. The unattended set contained distractors placed at random (new). In the unattended-old condition the unattended set of distractors (the set that did not contain the target) was placed in the same spatial configuration (old) in relation to the target that repeated across blocks. 
The attended set of distractors contained distractors placed at random (new). Finally, in the both-new condition each trial had both sets of distractors placed at random (new).

Participants completed the task with one of three ratios of attended to unattended stimuli, 75:25, 50:50, and 25:75 (see Fig. 1). One-third of participants were shown displays in which the ratio was 75:25: 11 attended distractors, 1 attended target, and 4 unattended distractors. A second group of participants was shown displays in which the ratio was 50:50: 7 attended distractors, 1 attended target, and 8 unattended distractors. Finally, a third group of participants was shown a display in which the ratio was 25:75: 3 attended distractors, 1 attended target, and 12 unattended distractors.

\section{Procedure}

After completing the consent/assent process, all adults and parents completed questionnaires addressing demographic information as well as a brief psychological and medical history. Adult participants received extra credit for a class for participating in the study. Child participants were told that in addition to a $\$ 5$ gift certificate, they could also earn "prizes" by finishing the epochs of the paradigm (described as "sets of pictures" to the children). For completing each epoch, children were awarded 5 points, earning a total of 30 for completing the entire task. They were told they could use these points to earn up to 2 toy prizes. However, all children were given two toys regardless of the number of epochs they completed. The behavioral requirements of the task were explained to participants just prior to the first epoch. In some cases, when it was not clear by the verbal or physical responses of the participant that they understood the task, comprehension was checked using a brief demonstration. Additional verbal feedback and clarification were provided during the beginning of the first block to children whose first few responses were incorrect. All participants were offered the opportunity to take a break after each block. Additionally, after the third block of trials participants were given a longer break so that the NEPSY visual attention task could be administered. ${ }^{1}$ All sessions

\footnotetext{
${ }^{1}$ The NEPSY Visual Attention Task (Korkman, Kirk, \& Kemp, 1998) was administered to all participants [however, a small subset of adults (3) completed the NEPSY before or after the context cueing task due to time constraints]. The NEPSY was given in the middle of the study to provide a structured break for child participants. The purpose of the NEPSY was to provide a standardized measure of attention that could be used to screen for participants with neurological disorders not otherwise determined by self report. As expected, results demonstrated a significant effect of age [one-way ANOVA, F $(1,38)=18.81, p<$ $.001]$ showing improvements in selective attention across development. However, no significant relevant correlations between the NEPSY visual attention task and the contextual cueing task were found. Thus, detailed findings from the NEPSY will not be presented.
}

with children were videotaped so that they could later be examined to determine if children were attending to the task. At the end of each session participants were queried concerning any awareness of repeating stimuli. To avoid false positives, participants were asked, "Did you notice anything special about the pictures?" Answers that were unclear were probed further using questions such as, "What do you mean by X?" At the end of the session children were given the opportunity to pick two small toys and were given the gift certificate. The entire session ranged from 25 min for adults completing the 25:75 task to one hour and 15 min for 10-year-olds completing the $75: 25$ task due to differences in the number of attended search items and differences in reaction times between adults and children.

\section{Results}

\section{Data analysis}

Both accuracy and reaction time data were analyzed. To ensure children were attending to the task, each session was videotaped (two were lost due to technical difficulties with the equipment). Videotapes of the children tested were coded to determine if children were attending to the task (attending in this case was defined as the child looking directly at the computer screen). No children were excluded based on this measure of attention as all qualifying participants showed attention to at least $90 \%$ of the trials (mean $=98.50, \mathrm{SD}=1.73$ ). Two 10-year-olds in each of the three tasks $(75: 25,50: 50$, and 25:75) reported awareness of repeating stimulus sets. Three adults in the $25: 75$ task, one adult in the 50:50 task, and five adults in the 75:25 task reported awareness of repeating stimulus sets. To ensure that including data from these participants did not alter findings, data were analyzed both including and excluding these participants. As analyses did not differ significantly, data from these participants were included in reported analyses.

Only data from correct responses were analyzed. Additionally, data were excluded when the reaction time was less than $300 \mathrm{~ms}^{2}$ or more than a cutoff level of two standard deviations (rounded to the nearest hundred milliseconds) above the mean reaction time for the age group (cutoffs: $8,700 \mathrm{~ms}$ for adults completing the $75: 25$ task, $5,500 \mathrm{~ms}$ for adults completing the 50:50 task, $2,400 \mathrm{~ms}$ for adults completing the $25: 75$ task, $11,100 \mathrm{~ms}$ for 10 year olds completing the 75:25 task, 7,200 ms for 10 year olds

\footnotetext{
${ }^{2}$ Responses faster than $300 \mathrm{~ms}$ were treated as either anticipations or responses to something other than the current stimulus, as this response time is faster than cognitive processing of the stimulus and response can occur.
} 
completing the 50:50 task, and 3,200 ms for 10 year olds completing the $25: 75$ task). This resulted in an average data loss of $3.58 \%(\mathrm{SD}=3.3)$.

A 2 (age) $\times 3$ (task) $\times 4$ (condition) $\times 6$ (epoch) ANOVA with age and task as a between-subjects factor and condition and epoch as within-subjects factors was performed on accuracy data. ${ }^{3}$ A 2 (age) $\times 3$ (task) $\times 4$ (condition) $\times 6$ (epoch) ANOVA with task as a betweensubjects factor and condition as a within-subjects factor was performed on reaction time data from the last epoch. Follow-up analyses were also performed with t-tests based on a priori hypotheses.

\section{Accuracy}

Accuracy data suggest improvements in performance across the study as a function of practice, as well as some possible differences as a function of age. Improvements in accuracy across epochs was demonstrated by a significant main effect of epoch $(\mathrm{F}(5,570)=5.38, \mathrm{p}=.002, \eta \mathrm{p} 2=.045)$. The absence of significant main effects of task or condition suggests these improvements are a function of practice. Additionally, while there was no significant main effect of age, there was a significant interaction between age and epoch $(\mathrm{F}(5,570)=3.17, \mathrm{p}=.027, \eta \mathrm{p} 2=.027)$. A follow-up ANOVA within each age group showed that the interaction of epoch and age was a result of the fact that epoch did not reach significance in adults, showing instead a trend effect $(\mathrm{F}(5,285)=2.16, \mathrm{p}=.066, \eta \mathrm{p} 2=.035)$. In contrast, children did show a main effect of epoch, demonstrating greater accuracy across the task $(\mathrm{F}(5,285)=5.09, \mathrm{p}=.006$, $\eta \mathrm{p} 2=.079)$. However, this improvement was limited as ceiling levels of accuracy were attained, with mean accuracy in all blocks for both adults and children above $94 \%$. No other significant interactions were found.

\section{Reaction time}

As can be seen in Figs. 2 and 3, reaction time data suggest that in addition to large reaction time differences between adults and children there are also differences in performance as a function of the task and condition. Both adults and children show learning in tasks with a greater number of repeating attended stimuli. Moreover, there were differences in contextual cueing based on the presence or absence of

\footnotetext{
${ }_{3}^{3}$ As reaction times varied between adults and children the data were transformed into z-scores (based on the overall reaction time for each age) in order to normalize overall reaction time across ages and confirm the main effect of condition and interaction between age and condition. This transformation did not alter these results, and all effects were in the same direction (all $p \mathrm{~s}<.05$ ). Additionally, age effects were confirmed using median rather than mean reaction times $(p \mathrm{~s}<.05)$.
}

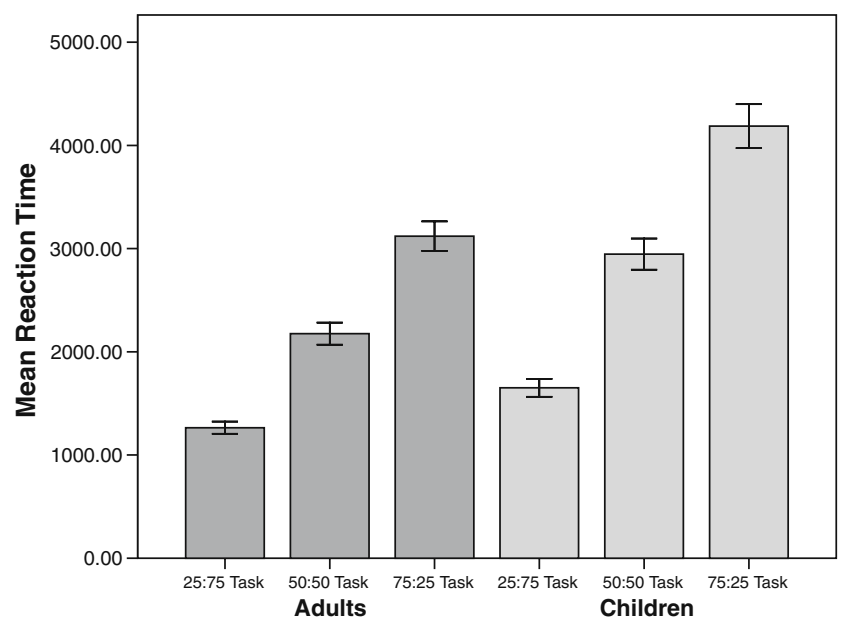

Fig. 2 Experiment 1. Mean reaction times (ms) for adults and children in the 25:75, 50:50, and 75:25 tasks

attention to repeated stimuli, and these contextual cueing effects differed as a function of age. These findings were demonstrated by main effects of age $(\mathrm{F}(1,114)=195.10, p<$ $\left..001, \eta_{\mathrm{p}}^{2}=.631\right)$, task $\left(\mathrm{F}(2,114)=570.70, p<.001, \eta_{\mathrm{p}}{ }^{2}=\right.$ $.909)$, condition $\left(\mathrm{F}(3,342)=6.91, p<.001, \eta_{\mathrm{p}}{ }^{2}=.057\right)$, and epoch $\left(\mathrm{F}(5,570)=77.06, p<.001, \eta_{\mathrm{p}}^{2}=.403\right)$. Additionally, there were significant interactions between age and task $\left(\mathrm{F}(2,114)=13.76, p<.001, \eta_{\mathrm{p}}{ }^{2}=.194\right)$, task and condition $\left(\mathrm{F}(6,342)=2.38, p=.029, \eta_{\mathrm{p}}{ }^{2}=.040\right)$, epoch and age $\left(\mathrm{F}(5,570)=6.35, p<.001, \eta_{\mathrm{p}}{ }^{2}=.053\right)$, task and epoch $\left(\mathrm{F}(10,570)=9.28, p<.001, \eta_{\mathrm{p}}{ }^{2}=.140\right)$, as well as between condition and epoch $(\mathrm{F}(15,1710)=2.88, p<.001$, $\left.\eta_{\mathrm{p}}{ }^{2}=.025\right)$. As the interaction between age and task suggests that adults and children show different patterns of learning as a function of the ratio of attended to unattended stimuli, follow-up analyses to explore other interactions were completed within each age group for each task.

Adults As anticipated, adults showed differences in contextual cueing as a function of the number of attended stimuli (i.e., task) with learning shown when the number of attended stimuli was high. Additionally there were practice effects across epochs and differences in learning as a function of attention. These findings were supported by significant main effects of task, condition, and epoch in adult reaction times $\left(\mathrm{F}(2,57)=320.03, p<.001, \eta_{\mathrm{p}}{ }^{2}=.918\right.$, $\mathrm{F}(3,171)=4.03, p=.008, \eta_{\mathrm{p}}^{2}=.066$, and $\mathrm{F}(5,285)=32$. $93, p<.001, \eta_{\mathrm{p}}^{2}=.366$ respectively). Additionally, there were significant interactions between task and epoch $(\mathrm{F}(10$, $\left.285)=6.13, p<.001, \eta_{\mathrm{p}}{ }^{2}=.177\right)$ as well as between condition and epoch $\left(\mathrm{F}(15,855)=1.93, p=.029, \eta_{\mathrm{p}}{ }^{2}=\right.$ .033). Follow-up t-tests on the overall reaction time within each task suggest the main effect of task is a function of the ratio of attended to unattended stimuli, with larger numbers of attended stimuli producing slower reaction times as a 

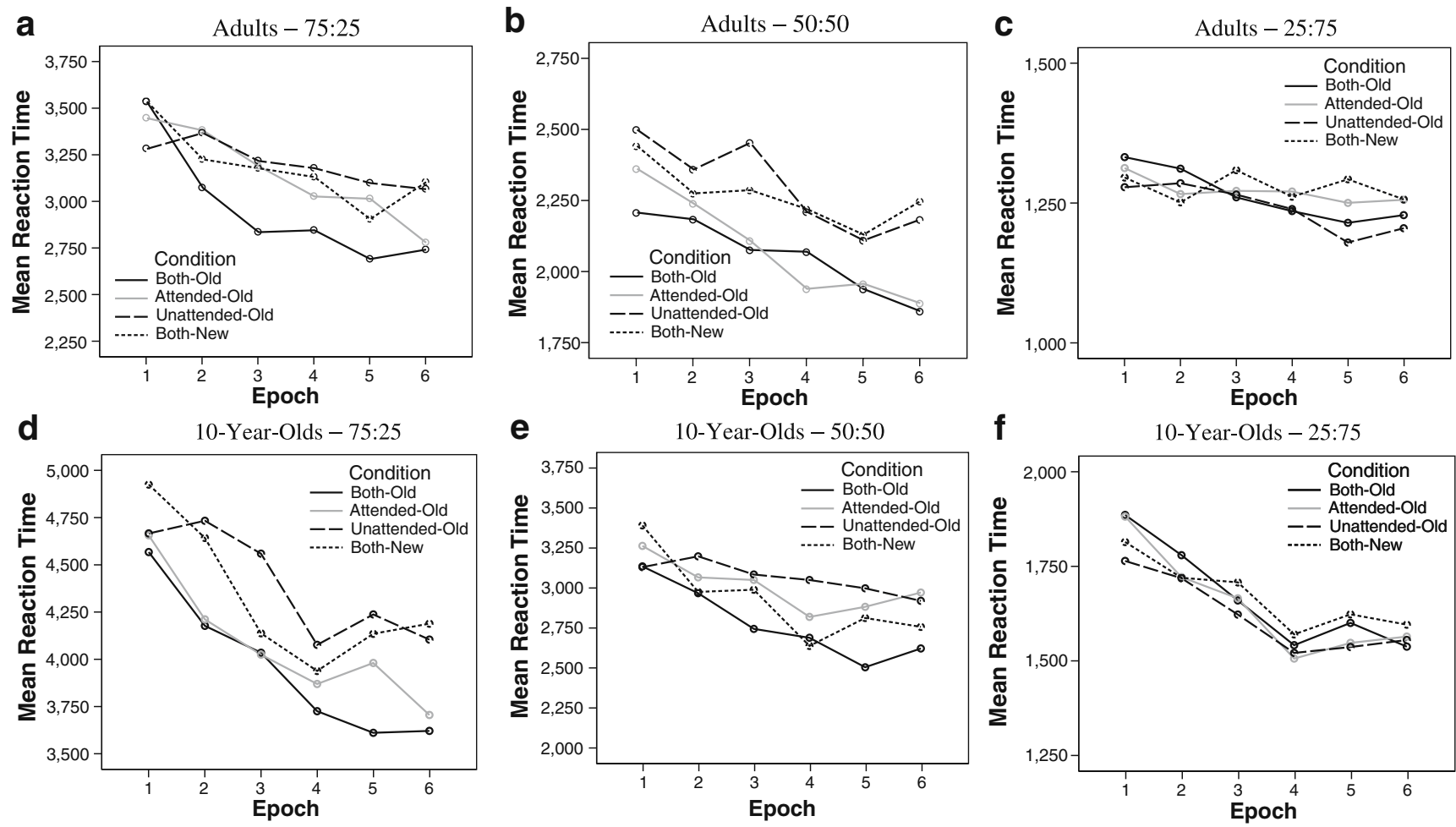

Fig. 3 Experiment 1. Reaction times (ms) by epoch. (a) Adult reaction times in the 25:75 task. (b) Adults reaction times in the 50:50 task (same as Fig. 2a). (c) Adult reaction times in the 75:25 task. (d) Ten year olds' reaction times in the 25:75 task. (e) Ten year olds'

consequence of effective selection (t-tests corrected for multiple comparisons: $25: 75$ vs. $50: 50 \mathrm{t}(38)=-15.61, p<$ $.001,50: 50$ vs. $75: 25 \mathrm{t}(38)=-11.08, p<.001$, and $25: 75$ vs. $75: 25 \mathrm{t}(38)=-25.10, p<.001$, see Fig. 2). Additional repeated measures ANOVAs were performed at each level of task to follow up the main effect of condition and epoch as well as their interactions (see Fig. 3).

Adults' 75:25 task As anticipated, adults showed a significant main effect of epoch $(\mathrm{F}(5,95)=15.03, \mathrm{p}<.001, \eta \mathrm{p} 2=$ .442) reflecting practice effects. Additionally, while there was not a significant main effect of condition, there was a significant interaction between condition and epoch $(\mathrm{F}(15$, $285)=1.70, \mathrm{p}=.049, \eta \mathrm{p} 2=.078)$. T-tests comparing condition in the first and last epoch confirm learning effects in the two critical conditions. There were no significant differences between conditions in the first epoch, but reaction times were significantly faster for the both-old and attended-old conditions compared to the both-new condition in the final epoch $(\mathrm{t}(19)=-3.09, \mathrm{p}=.006$ and $\mathrm{t}(19)=-2.13, \mathrm{p}=.047$, respectively). No difference in reaction time was found between the unattended-old and both-new conditions in the final epoch. Additional exploratory comparisons of the critical conditions in the remaining epochs (2 through 5) suggest significant learning effects can reaction times in the 50:50 task (same as Fig. 2b). (f) Ten year olds' reaction times in the 75:25 task. Please note that Y-axes differ between figures due to large differences in reaction times between tasks and across ages

be seen in the both-old condition as early as the third block $(\mathrm{t}(19)=-2.52, \mathrm{p}=.021)$; however, it is not seen in the attended-old condition until the final block (all ps $>.05$ ). One possibility as to why learning of the attended-old condition did not appear until the final epoch is that three participants, while not outliers, showed significantly slower reaction times in the fifth epoch as compared to all other epochs creating noise in the data. If these particular participants were removed from analyses, performance on the $75: 25$ task was similar to that of adults on the 50:50 task.

Adults' 50:50 task As in the original Jiang and Chun (2001) study, adults showed a significant main effect of epoch and condition $(F(5,95)=17.10, \mathrm{p}<.001, \eta \mathrm{p} 2=.474$ and $\mathrm{F}(3,57)=4.00, \mathrm{p}=.015, \eta \mathrm{p} 2=.174$, respectively). Additionally there was a significant interaction between condition and epoch $(\mathrm{F}(15,285)=1.79, \mathrm{p}=.036, \eta \mathrm{p} 2=$ .086). Learning was confirmed by t-tests at the first and last epochs. There were no significant differences between conditions in the first epoch, but significantly faster reaction times for the both-old and attended-old conditions compared to the both-new condition in the final epoch $(\mathrm{t}(19)=-3.19, \mathrm{p}=.005$ and $\mathrm{t}(19)=-4.16, \mathrm{p}=.001$, respectively) as well as no significant differences between 
the unattended-old and both-new conditions. Additional exploratory comparisons of the critical conditions in the remaining epochs (2 through 5) suggest significant learning effects can be seen in the both-old condition as early as the fourth block $(\mathrm{t}(19)=-3.29, \mathrm{p}=.004)$; however, it is not seen in the attended-old condition until the final block (all ps $>.05$ ).

Adults' 25:75 task There was a significant effect of epoch for this task $(\mathrm{F}(5,95)=3.99, \mathrm{p}=.002, \eta \mathrm{p} 2=.174)$, but no significant main effect of condition or significant interactions ( $p$ 's $>.05$ ), suggesting no learning occurred.

Ten year olds As in adult data, in addition to practice effects, children showed greater learning when the number of attended stimuli was high, and performance differed as a function of attention. However, children required a higher number of attended stimuli to show learning as compared to adults. These findings were supported by significant main effects of task, condition, and epoch $(\mathrm{F}(2,57)=279.24$, $\mathrm{p}<$ $.001, \eta \mathrm{p} 2=.907, \mathrm{~F}(3,171)=3.32, \mathrm{p}=.021, \eta \mathrm{p} 2=.055$, and $\mathrm{F}(5,285)=46.07, \mathrm{p}<.001, \eta \mathrm{p} 2=.447$, respectively). Additionally, there were significant interactions between task and epoch $(\mathrm{F}(10,285)=4.37, \mathrm{p}<.001, \eta \mathrm{p} 2=.133)$ as well as between condition and epoch $(\mathrm{F}(15,855)=1.98$, $\mathrm{p}=.019, \eta \mathrm{p} 2=.034)$. Follow-up tests on mean reaction time suggest the main effect of task is a function of the number of stimuli, with larger numbers of attended stimuli producing slower reaction times as a consequence of effective selection (t-tests corrected for multiple comparisons: $25: 75$ vs. $50: 50 \mathrm{t}(38)=-15.55, \mathrm{p}<.001,50: 50$ vs. $75: 25 \mathrm{t}(38)=-9.95, \mathrm{p}<.001$, and $25: 75$ vs. $75: 25, \mathrm{t}(38)=$ $-23.09, \mathrm{p}<.001$, see Fig. 2). Additional repeated measures ANOVAs were performed at each level of the task to follow up the main effects of condition and epoch as well as their interactions (see Fig. 3).

Ten year olds' 75:25 task In the 75:25 task 10 year olds, like adults, showed a main effect of epoch $(\mathrm{F}(5,95)=$ $19.52, \mathrm{p}<.001, \eta \mathrm{p} 2=.507)$. They also showed a main effect of condition $(\mathrm{F}(3,57)=3.17, \mathrm{p}=.031, \eta \mathrm{p} 2=.143)$, but did not show a significant interaction between the two $(p>.05)$. However, learning was seen when looking at the first as compared to the final epoch, demonstrating no significant differences between conditions in the first epoch, but faster reaction times for the both-old and attended-old conditions compared to the both-new condition in the final epoch $(\mathrm{t}(19)=-2.74, \mathrm{p}=.013$ and $\mathrm{t}(19)=$ $-2.31, p=.032$, respectively). Additionally, there was no significant difference between attended-new and both-new conditions. These findings are similar to those found in the adult 75:25 and 50:50 tasks. Additional exploratory comparisons of the critical conditions in the remaining epochs (2 through 5) suggest significant learning effects can be seen in the both-old and attended-old conditions as early as the second epoch $(\mathrm{t}(19)=-2.79, \mathrm{p}=.012$ and $\mathrm{t}(19)=-2.31, \mathrm{p}=.032$ respectively). Interestingly, there was also an unexpected significant difference between the unattended-old and both-new conditions in the third epoch $(\mathrm{t}(19)=2.44, \mathrm{p}=.026)$ reflecting significantly slower reaction times for unattended-old displays (i.e., not learning effects); however, this did not remain across epochs (all other ps $>.05$ ).

Ten year olds' 50:50 task Ten year olds in this task did show a significant effect of epoch $(\mathrm{F}(5,95)=13.44$, $\mathrm{p}<.001, \eta \mathrm{p} 2=.414)$ as well as a significant interaction between condition and epoch $(\mathrm{F}(15,285)=1.85, \mathrm{p}=.035$, $\eta \mathrm{p} 2=.089)$. However, no main effect of condition was seen in this task, and follow-up comparisons at the first and last epoch did not show differences between conditions. This suggests that while there may be some learning present, it is not strong enough to be seen by the final epoch. Moreover, additional exploratory comparisons of the critical conditions in the remaining epochs ( 2 through 5) do not show significant learning effects in either the both-old condition or attended-old condition. However, similar to the 75:52 task, there was an unexpected significant difference between the unattended-old and both-new conditions in the fourth epoch $(\mathrm{t}(19)=2.29$, $\mathrm{p}=.034)$ reflecting significantly slower reaction times for unattended-old displays; however, this did not remain across epochs (all ps $>.05$ ).

Ten year olds' $25: 75$ task Finally, similar to adults on the 25:75 task, there was a significant effect of epoch in the $25: 75$ task $(\mathrm{F}(5,95)=26.22, \mathrm{p}<.001, \eta \mathrm{p} 2=.58)$ reflecting practice effects. However, there was no main effect of condition or interactions suggesting no learning occurred.

\section{Discussion}

The results of Experiment 1 demonstrate that 10 year-olds, like adults, show faster reaction times when searching through 4 as compared to 12 stimuli in the relevant color, thereby demonstrating effective attentional guidance by color. However, post-hoc analyses examining regression coefficients to compare reaction time changes as a function of the number of search items in relation to age shows significant differences in the rate of change between adults and children $(\mathrm{t}=-5.27, \mathrm{p}<.001)$ as children in this task took on average $107 \mathrm{~ms}(\mathrm{SD}=18.9)$ more for each additional search item. This may be a result of differences in selection across age, but is more likely a result of large 
differences in reaction times as a function of motor development (both hand and eye movements) that would influence the rate of change (e.g., Eckert \& Eichorn, 1977), as well as reflect differences in the strategies children use during visual search tasks (Donnelly, Cave, \& Geenway, 2007)

Learning in children (as reflected in an interaction between condition and epoch and/or significant differences between conditions in reaction times in the final epoch) varied as a function of the ratio of attended to unattended stimuli, suggesting that while attentional guidance by color is efficient and contextual cueing is possible in 10 year olds, an additional factor impedes performance on this task. Children learned when the ratio of attended to unattended stimuli was high, but not when this ratio was $50: 50$ or lower (i.e., in the 25:75 task). The data show that both adults and children demonstrate learning of the both-old and attended old conditions in the $75: 25$ task as indicated by significant differences in reaction times at the last epoch. However, in the 50:50 task, adults show learning of the both-old and unattended-old condition, whereas children do not (although a significant interaction between epoch and condition in children may reflect weak learning in the bothold, but not the attended-old condition as seen in Fig. 3e). Finally, neither adults nor 10 year olds show significant learning in the 25:75 task. One possible explanation of the absence of learning in the $25: 75$ condition is that the search task was so easy in this condition that there was little opportunity for learners to show improvement (i.e., both groups may be performing at ceiling levels) or that the benefits of learning were not significant enough to induce learning. A second possibility was that the small number of unattended items made them more salient, resulting in attentional capture by these items and in turn reducing contextual cueing such as has been seen in a recent study by Conci and von Mühlenen (2009).

A potential limitation of this experiment was that in order to examine the development of selection, the total number of items of the display was held constant while the number of attended items was varied. Although this allowed for the isolation of attentional guidance by color, there are two potential interpretations of the learning effects. One possible explanation for learning effects seen in the 75:25 task is that this condition had fewer unattended distractors, thus decreasing the level of distraction and allowing for greater learning. This explanation is consistent with studies that show general improvements in filtering across development (e.g., Enns \& Akhtar, 1989). However, another explanation is also possible. Recent studies suggest that the ability to filter out unattended distractors improves with greater perceptual load (Forster \& Lavie, 2007) and that the level of perceptual load needed for sufficient selection and filtering changes across development (Huang-
Pollock, Carr, \& Nigg, 2002). Vaidja et al. (2007) argue that the attentional immaturity potentially responsible for lack of learning in children could be related to children's employment of early attentional systems at lower levels of perceptual load as compared to adults. In the Vaidja et al. task this would result in reduced attention to all distractors as there was only one set of distractors, whereas in the task utilized in this study as there are two sets of distractors (attended and unattended) this could conceivably assist in reducing attention to the unattended distractors and assist with learning the context created by the attended distractors. Specifically, the increase in attended distractor stimuli from 8 to 12 in the $75: 25$ task increases the attended perceptual load and therefore may also facilitate filtering of the unattended distractors. Thus, to examine if improvements in learning seen in the 75:25 condition were due to increased perceptual load rather than a reduced number of distractors, a second experiment examined the role of perceptual load in this task.

\section{Experiment 2}

Recent studies have suggested that an increase in perceptual load may improve the ability to filter out unwanted information (Huang-Pollock et al., 2002; Lavie, Hirst, de Fockert, \& Viding, 2004). Perceptual load in this study is defined as the total amount of potentially task-relevant information available (and attended to) in the external environment (Lavie, 1995; Huang-Pollock et al., 2002). For example, Huang-Pollock et al. (2002) asked children and young adults to search displays of different set sizes flanked by irrelevant distractors. Children's performance was similar to adults when perceptual load (i.e., set size) was high but was reduced when perceptual load was low. Therefore, Experiment 2 manipulated perceptual load by increasing perceptual load within the stimulus display. To increase perceptual load without altering the ratio of unattended to attended distractors, this ratio remained at 50:50 while the total number of stimuli was increased from 16 to 24 . This was then compared to the $75: 25$ and the 50:50 tasks from Experiment 1, which contained only 16 stimuli. It is hypothesized that if improvements in performance in the $75: 25$ task with 16 stimuli were a result of increased perceptual load (i.e., searching through 12 attended distractors in comparison to 8 in the 50:50 task), then children's performance in the contextual cueing paradigm with 24 distractors (12 attended 12 unattended) should be similar to that on the 75:25 task with 16 stimuli, showing learning of the both-old condition and the attended-old conditions. In contrast, if improvements in performance were a result of the reduced number of distractors in the 75:25 task with 
16 stimuli, then performance on the 50:50 task with 24 stimuli should not improve and no evidence of learning should be found.

\section{Method}

Participants

Sixty 10 year olds participated in this experiment. As in Experiments 1, the number of participants was selected based on previous studies. Results from 40 10-year-old children were taken from Experiment 1 as their data reflect two of the three tasks relevant to this experiment (i.e., the 50:50 task with 16 stimuli and the 75:25 task with 16 stimuli, respectively). The remaining participants consisted of 23 10-year-old children. Two participants were excluded due to physical, psychological, neurological, or learning disorders. Thus, the final analyses included 21 additional 10 year olds ( 10 female, 11 male, 19 Caucasian, 1 Asian, and 1 mixed-heritage, mean age $=10$ years 7 months, $\mathrm{SD}=$ 2.77 months). The participants for the 50:50 task with 24 stimuli were recruited from the Minneapolis area as well as from the Pioneer Valley area of Western Massachusetts and were screened in the same way as in Experiment 1. Additionally, the new participants were compensated with $\$ 10$ due to the increased length of time to complete the task, and 14 parents were compensated with $\$ 5$ for traveling expenses. As in Experiment 1, parents of children gave written consent, and all 10-year-old children gave written assent, in accordance with the University of Minnesota and Hampshire College Institutional Review Boards.

\section{Stimuli and design}

Data were collected from one new task in Experiment 2. The stimuli were the same as those used in Experiment 1 , and the ratio of attended to unattended distractors in this task was 50:50. However, in contrast to Experiment 1 , in which displays contained 16 total stimuli, the new task contained a total of 24 stimuli. Data from Experiment 1 (the 50:50 and 75:25 task with 16 total stimuli) were then compared to data from the new 50:50 task with 24 stimuli.

\section{Procedure}

The procedure for this experiment was the same as in Experiment 1. However, the study duration increased to a maximum of $1 \mathrm{~h}$ and $15 \mathrm{~min}$ for the 50:50 task with 24 stimuli due to the increase in the number of attended search items. Additionally, the NEPSY was not conducted, and although all sessions were videotaped, attention behavior was not coded in this task. ${ }^{4}$

\section{Results}

Data analysis

Both accuracy and reaction time data were analyzed. As in Experiment 1, only correct responses were analyzed, and data were excluded if the response time was less than $300 \mathrm{~ms}$ or more than two standard deviations above the mean reaction time (cutoffs: $7,200 \mathrm{~ms}$ for 10 year olds completing the 50:50 task with 16 distractors, $12,100 \mathrm{~ms}$ for 10 year olds completing the 50:50 task with 24 distractors, and $11,100 \mathrm{~ms}$ for 10 year olds completing the 75:25 task with 16 distractors). ${ }^{5}$ This resulted in an average data loss of $4.17 \%(\mathrm{SD}=2.86)$. Only one participant in the 50:50 task with 24 stimuli reported any explicit awareness. Therefore, as in Experiment 1 this participant was retained in reported analyses.

A 3 (task) $\times 4$ (condition) $\times 6$ (epoch) ANOVA with task as a between-subjects factor and condition and epoch as within-subjects factors was performed on accuracy and reaction time data. Follow-up analyses were performed as in Experiment 1 to examine any significant interactions based on a priori hypotheses.

Accuracy As in Experiment 1, there were practice effects, showing particular improvements in the 50:50 task with 24 stimuli. These findings were supported by a significant main effect of epoch $(\mathrm{F}(5,285)=4.59, \mathrm{p}=$ $.012, \eta \mathrm{p} 2=.075)$ reflecting greater accuracy across epochs. Additionally, there was a significant interaction among task, condition, and epoch $(\mathrm{F}(30,855)=1.58$, $\mathrm{p}=.037, \eta \mathrm{p} 2=.053)$ reflecting greater improvements in accuracy for children across the first two epochs in the 50:50 task with 24 stimuli, averaging 94.54 in the first epoch and 98.26 in the second. Overall, accuracy was high, averaging 97.74 (SD 2.47) across epochs and conditions.

\footnotetext{
${ }^{4}$ The NEPSY was not completed because the previous experiment suggested that this measure was not correlated with behavior on the contextual cueing paradigm. Additionally, while each participant's session was videotaped these tapes were not coded for attention to the task because the previous experiment showed that 10 year olds attended to more than $90 \%$ of trials on the contextual cueing paradigm.

${ }^{5}$ The difference in the cutoff between the 50:50 task with 24 stimuli as compared to the $75: 25$ task with 16 stimuli was based primarily on differences in the standard deviation, which was greater in the 50:50 task with 24 distractors (mean RT for 50:50 with 24 stimuli $=4,775$, mean RT for $75: 25$ with 16 stimuli $=4,595$ ).
} 
Reaction time As in Experiment 1, there was an increase in reaction times as the number of attended stimuli increased (i.e., 8 or 12). Moreover, there were practice effects across epochs as well as a main effect of condition suggesting similar patterns in performance across tasks. Differences in reaction time as a function of the number of attended stimuli were supported by a significant main effect of task $(\mathrm{F}(2,58)=59.15, \mathrm{p}<.001, \eta \mathrm{p} 2=.671)$. Follow-up t-tests on overall mean reaction times in each task indicate that reaction times were faster for the 50:50 task with 16 stimuli ( 8 attended stimuli) as compared to both the 75:25 task with 16 stimuli (12 attended stimuli) $(\mathrm{t}(38)=-9.95, \mathrm{p}<.001)$ and 50:50 task with 24 stimuli (12 attended stimuli) $(\mathrm{t}(39)=$ $-10.01, \mathrm{p}<.001)$. Moreover, there were no significant differences in reaction time between the 75:25 task with 16 stimuli and the 50:50 task with 24 stimuli ( $>>75$ ), which both contained 12 attended stimuli. In addition to the main effect of task, there was a significant main effect of epoch $(\mathrm{F}(5,290)=44.16, \mathrm{p}<.001, \eta \mathrm{p} 2=.432)$ reflecting practice effects, and a main effect of condition $(\mathrm{F}(3,174)=2.92$, $\mathrm{p}=.036, \eta \mathrm{p} 2=.048)$. Follow-up t-tests suggest the main effect of condition is driven by significantly faster reaction times to the both-old as compared to both-new condition $(t(60)=-3.36, p=.001)$ in the final epoch while there were no significant differences in the first epoch. Additionally, there were no significant differences between both-new and the other two conditions (attended-old and unattended-old) at either the first or final epoch.

While there was a significant interaction between task and epoch $(\mathrm{F}(10,290)=2.84, \mathrm{p}=.003, \eta \mathrm{p} 2=.089)$, and condition and epoch $(\mathrm{F}(15,870)=2.23, \mathrm{p}=.008, \eta \mathrm{p} 2=$ $.037)$, there was no significant interaction between condition and task. However, to explore a priori hypotheses regarding differences between conditions within each task, a repeated measures ANOVA was performed on the data from the 50:50 task with 24 stimuli.

50:50 task with 24 stimuli As can be seen in Fig. 4, while children showed practice effects, significant learning did not occur in this task. There was a significant main effect of epoch in this task $(\mathrm{F}(5,100)=16.12, \mathrm{p}<.001, \eta \mathrm{p} 2=.446)$, but no significant main effect of condition or interaction between epoch and condition in the 50:50 task with 24 stimuli (see Fig. 4). However, as a main effect of condition was found in the omnibus ANOVA, follow-up t-tests were conducted to explore the possibility that effects at the final epoch are contributing to the omnibus main effect. T-tests revealed that while there were no significant effects at either the first or final epoch, there was a marginally significant difference between the both-old and both-new condition at the final epoch $(\mathrm{t}(19)=-1.89, \mathrm{p}=.074)$. Additionally exploratory comparisons of the critical conditions in the remaining epochs (2 through 5 ) found one
10-Year-Olds: 50:50 with 24 Stimuli

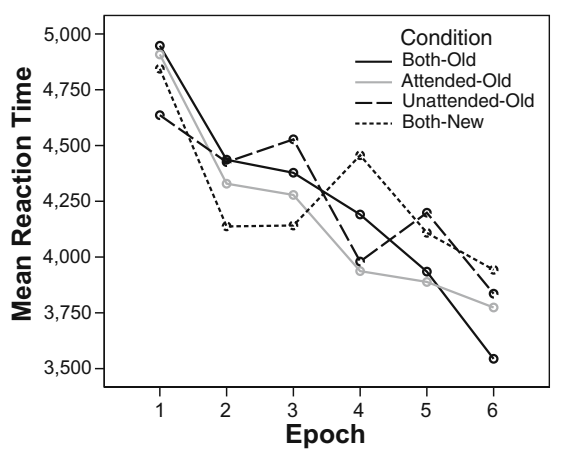

Fig. 4 Experiment 2. Reaction times (ms) by epoch. Ten year olds' reaction times in 50:50 task with 24 stimuli (for 10 year olds' reaction times to 50:50 task with 16 stimuli, see Fig. 1e; for 75:25 task with 16 stimuli, see Fig. 1d)

additional significant difference in the fourth epoch between the attended-old and both-new condition $(\mathrm{t}(19)=$ $-2.56, \mathrm{p}=.019$ ); however, this difference did not appear in later epochs (all ps $>.05$ ). While this likely contributed to the overall main effect of condition and may suggest some benefit of increasing perceptual load, the effect was not large enough to produce significant learning in this task. Thus, findings are consistent with the interpretation that increased numbers of attended stimuli (i.e., increased perceptual load) did not significantly improve filtering aspects of performance on the contextual cueing task used in this study.

\section{Discussion}

Increasing the perceptual load by increasing the total number of attended distractor stimuli did not improve performance on the contextual cueing paradigm. While reaction times increased due to the increased number of attended stimuli, suggesting relatively mature attentional guidance by color (replicating findings from Experiment 1), reaction times did not show significant learning at the last epoch. However, like the 50:50 task with 16 stimuli, the 50:50 task with 24 stimuli showed some hints that learning of the both-old condition was present (evidenced by a nonsignificant trend difference between the both-old and bothnew conditions at the final epoch). These results do not support an increased perceptual load interpretation of learning effects seen in the 75:25 task as performance in this task did not improve. One possible explanation for the absence of improvements in learning could be that the increased number of items (24 as compared to 16) reduced the possible context variability. However, as there were 96 possible locations, it is unlikely this is the primary reason that perceptual load did not improve learning. Instead 
results suggest it is a reduced need to filter out unattended distractors that facilitates learning in the 10-year-old children or alternatively that the added dimension of color impairs contextual cueing in this task.

\section{General discussion}

Findings from these experiments suggest that while attentional guidance by color is relatively mature by the age of 10, implicit learning in the contextual cueing paradigm is unreliable. Implicit learning was expressed when there were a high number of relevant attended distractors and few unattended distractors (i.e., the 75:25 task), but not when the number of unattended distractors was similar or greater than the number of attended distractors (i.e., the 50:50 and 25:75 task).

Both Experiments 1 and 2 demonstrate that by the age of 10 , children are able to use attentional guidance by color to effectively attend to the relevant context (i.e., the attended color). Results show significantly increasing reaction times as a function of the number of attended stimuli (from 4 in the 25:75 task to 12 in the 75:25 task) for both adults and children. Findings were replicated in Experiment 2 when the total number of distractor stimuli was raised to 24 . In Experiment 2, reaction times were slower for the 75:25 task with 16 stimuli (12 attended) and 50:50 task with 24 distractor stimuli (12 attended) as compared to the 50:50 task with 16 distractor stimuli ( 8 attended). However, there was no difference in reaction time between the 75:25 task with 16 stimuli and the 50:50 task with 24 distractor stimuli as both tasks contained 12 attended distractors. These reaction time findings reflect relatively mature attentional guidance by color and replicate visual search studies with adults that show that during serial search tasks such as this, each additional search item increases reaction time (for a review, see Palmer, Verghese, \& Pavel, 2000). They also replicate findings in children that show increased reaction times with increased set sizes (e.g., Donnelly et al., 2007) and extend them to show that this process is effective in the presence of distractors. Nevertheless, the findings from this study do not exclude the development of attentional selection processes. It is possible that with a more difficult selection task developmental differences may be found. While effective attentional guidance by color in the presence of distractors suggests that this aspect of attention is not primary factor in unreliable implicit learning in this task, there are several other possibilities that remain.

First, it is possible that implicit learning is not sufficiently functional in 10 year olds to allow for the expression of learning in the contextual cueing paradigm. While the majority of previous research suggests that there is little development of implicit learning across childhood (e.g., Don, Schellenberg, Reber, DiGirolamo, \& Wang, 2003; Meulemans et al., 1998; Thomas \& Nelson, 2001; Thomas et al., 2004), there have been a few implicit learning studies that have suggested developmental differences (Maybery, Taylor, \& O’Brien-Malone, 1995; Thomas \& Nelson, 2001; Thomas et al., 2004; Vaidya et al., 2007). Thus, in the experiments presented in this study it is possible that developing implicit learning is contributing to overall learning. However, any contribution of this development does not fully explain the findings. For example, if children required more total relevant information for implicit learning to occur, increased information presented in the 50:50 task with 24 stimuli should have improved learning of the both-old and attended-old conditions as it contained the same number of attended distractor stimuli as the 75:25 task. However, this was not the case. Instead, learning was lost, suggesting interference from distractors in this task. Another difference between the task used in this study and previous adult studies is that only three displays of each condition type were included rather than six as in previous studies. This was done to reduce the time of the study as children have a shorter attention span as compared to adults. However, this also reduced the number of displays contributing to reaction time differences. For example, children in the 50:50 task showed a significant interaction between condition and epoch but no significant differences at the final epoch. It is possible that children had weak learning of the both-old condition, but the reduced number of displays made this difficult to see (see Fig. 3e). While learning is typically seen after just 3 to 5 repetitions, we attempted to account for the reduced number of displays by examining learning at the final epoch as has been done in other studies (e.g., Chun \& Jiang, 2003). Additionally, as implicit learning was seen in adults and in children under some circumstances, it is unlikely that this is the primary reason implicit learning was not seen reliably in children. However, as learning was not as strong in children as compared to adults in this study, future research should continue to explore possible contributions of the development of implicit learning. Moreover, as this study focused on one age group, the inclusion of children both younger and older than those included in this study would help to map out the development of implicit learning in this task.

Second, an assumption held in this study is that learning in this task is implicit rather than explicit. Chun and Jiang (1998) demonstrated that in the context cueing task explicit awareness of repetition does not correlate with the magnitude of learning. Moreover, they suggest that it is implicit rather than explicit learning that accounts for performance in the context cueing task. However, while only a small number of participants reported explicit awareness of some form in this study, it is possible that 
children, unlike adults, employ explicit learning as a strategy in this task. If children do employ this strategy, it is possible that development of explicit learning contributes to reduced performance in children. However, as the use of explicit learning was not assessed, this should be studied further to ensure that like adults, explicit learning and awareness do not contribute to learning in this task in children.

Third, in Experiment 1, adults and children showed similar performance when there were a greater number of relevant attended distractors as compared to unattended distractors (75:25 task), but not when there were equal numbers of attended and unattended stimuli. In the 50:50 task adults succeed in learning and children fail to learn (although it is possible that while not significant at the final epoch, there was learning of the both-old condition for children). Moreover, both adults and children do not show learning when there are few attended distractors (75:25 task). One possible explanation of this pattern is that the level of perceptual load influences the ability to effectively show contextual cueing, thus Experiment 2 was designed to examine this possibility. As noted in earlier discussions, across development, the optimal level of perceptual load is likely to change (HuangPollock et al., 2002). However, results from Experiment 2 do not support this interpretation, as increasing perceptual load in the contextual cueing paradigm used here did not improve children's performance. Instead, it is likely that improved performance among children in the 75:25 task with 16 stimuli resulted from fewer distractors. While previous studies suggest that the number of participants (20 in each task) should have provided sufficient power to detect learning effects in the 50:50 task with 24 stimuli (e.g., Jiang \& Chun, 2001; Dixon et al., 2010), future studies should explore these findings further.

Fourth, while beyond the scope of this study, the added dimension of color may be an important factor in contextual cueing for children. For example, it is possible that a contextual cueing task containing only the both-old and both-new conditions but utilizing multi-colored distractor stimuli (i.e., all distractors are relevant) may be more difficult for children as compared to when distractor stimuli are all of the same color. However, results from Dixon et al. (2010), which utilized multi-colored stimuli (red and green), suggest that if anything, color may contribute to learning by adding additional contextual features. Moreover, as children did show learning in the 75:25 task (and may have some level of learning in the 50:50 task), it is likely other factors contribute more strongly to effects seen in this study.

Finally, as mentioned in the introduction, selective attention consists of multiple processes. Most studies focus on two common skills; the ability to allocate attention selectively to one aspect of a display or stimulus, and the ability to ignore irrelevant aspects of the display or stimulus. In the developmental literature the term selection refers to the former process and filtering to the latter (e.g., Akhtar \& Enns, 1989; Ridderinkhoff \& van der Stelt, 2000). In contrast, the terms enhancement/facilitation and inhibition/suppression are often used in the adult literature to refer to similar concepts (e.g., Mangun \& Hillyard, 1991; Awh, Matsukura, \& Serences, 2003). Traditionally, selection and filtering have been thought of as two aspects of one process such that the selection of stimuli in the environment necessitates the filtering of the remaining stimuli. However, studies of selective attention in adults suggest that under certain conditions, non-selected material is processed despite efficient selection (e.g., Chun \& Jiang, 1998). Moreover, studies of the neural underpinnings of selective attention suggest it involves both relative enhancement (i.e., selection) of the signal of the attended stimulus (e.g., Di Russo, Martinez, \& Hillyard, 2003; Handy \& Khoe, 2005; Hillyard, Vogel, \& Luck, 1998; Mangun \& Fannon, 2007; Mangun \& Hillyard, 1991, 1995) as well as relative suppression (i.e., filtering) of the unattended stimulus (e.g., Awh et al., 2003; Caputo \& Guerra, 1998; Dell'Acqua, Pesciarelli, Jolicoeur, Eimer, \& Peressotti, 2007; Serences, Yantis, Culberson, \& Awh, 2004). Moreover, recent research suggests that these may be separable processes (e.g., Slotnick, Schwarzenback, \& Yantis, 2003; Tipper et al., 1997). Thus, selection (i.e., facilitation/enhancement) and filtering (i.e., inhibition/ suppression) may be two complementary, interacting processes with partially shared or possibly even distinct underlying mechanisms. Moreover, if this is the case, selection and filtering systems may have different developmental trajectories. In this study attentional guidance by color can be equated with selection as it is critical to select the appropriate color to guide attention in the visual search task. However, filtering of the unattended distractors is also required for efficient learning to take place. While there is evidence that filtering is present in young infants (e.g., Newman \& Jusczyk, 1996), it improves across childhood. For example, Enns and Akhtar (1989) asked children (ages 4-7) and adults to perform a flanker task where performance is related to the ability to ignore distracting stimuli (i.e., filtering). Findings from this study and others (e.g., Rueda et al., 2004; McDermott et al., 2007) suggest that children are less able to ignore irrelevant flanking stimuli as compared to adults. As performance improved with a reduction in the number of distractors it is possible that relative immaturity of this aspect of selective attention may have contributed to the absence of learning when more distractors were present. Moreover, it raises the intriguing possibility of different developmental trajectories for these two aspects of selective attention.

This study highlights important factors in the relationship between selective attention and implicit learning 
within the context cueing paradigm. This study demonstrates that, while attentional guidance by color is relatively mature by the age of 10 , implicit learning, while present under some conditions, is unreliable. In particular, the number of unattended distractors appears to play an important role in the expression of implicit learning.

\section{References}

Akhtar, N., \& Enns, J. T. (1989). Relations between covert orienting and filtering in the development of visual attention. Journal of Experimental Child Psychology, 48(2), 315-334. doi:10.1016/ 0022-0965(89)90008-8

Awh, E., Matsukura, M., \& Serences, J. T. (2003). Top-down control over biased competition during covert spatial orienting. Journal of Experimental Psychology: Human Perception and Performance, 29(1), 52-63. doi:10.1037/0096-1523.29.1.52

Caputo, G., \& Guerra, S. (1998). Attentional selection by distractor suppression. Vision Research, 38(5), 669-689. doi:10.1016/ S0042-6989(97)00189-2

Chun, M. M., \& Jiang, Y. (1998). Contextual cueing: Implicit learning and memory of visual context guides spatial attention. Cognitive Psychology, 36, 28-71. doi:10.1006/cogp.1998.0681

Chun, M. M., \& Jiang, Y. (2003). Implicit, long-term spatial contextual memory. Journal of Experimental Psychology. Learning, Memory, and Cognition, 29(2), 224-234. doi:10.1037/0278-7393.29.2.224

Cohen, A., Ivry, R. I., \& Keele, S. W. (1990). Attention and structure in sequence learning. Journal of Experimental Psychology. Learning, Memory, and Cognition, 16(1), 17-30. doi:10.1037/ 0278-7393.16.1.17

Columbo, J. (2001). The development of visual attention in infancy. Annual Review of Psychology, 52, 337-367. doi:10.1146/annurev. psych.52.1.337

Conci, M., \& von Mühlenen, A. (2009). Region segmentation and contextual cuing in visual search. Attention, Perception, \& Psychophysics, 71(7), 1514-1524. doi:10.3758/APP.71.7.1514

Dell'Acqua, R., Pesciarelli, F., Jolicoeur, P., Eimer, M., \& Peressotti, F. (2007). The interdependence of spatial attention and lexical access as revealed by early asymmetries in occipito-parietal ERP activity. Psychophysiology, 44(3), 436-443. doi:10.1111/j.14698986.2007.00514.x

Di Russo, F., Martinez, A., \& Hillyard, S. A. (2003). Source analysis of event-related cortical activity during visual-spatial attention. Cerebral Cortex, 13, 486-499. doi:10.1093/cercor/13.5.486

Dixon, M. L., Zelazo, P. D., \& De Rosa, E. (2010). Evidence for intact memory-guided attention in school-aged children. Developmental Science, 13(1), 161-169. doi:10.1111/j.1467-7687.2009.00875.x

Don, A. J., Schellenberg, E. G., Reber, A. S., DiGirolamo, K. M., \& Wang, P. P. (2003). Implicit learning in children and adults with Williams syndrome. Developmental Neuropsychology, 23, 201225. doi:10.1207/S15326942DN231\&2 9

Donnelly, N., Cave, K., \& Greenway, R. (2007). Visual search in children and adults: Top-down and bottom-up mechanisms. The Quarterly Journal of Experimental Psychology, 60(1), 120-136. doi:10.1080/17470210600625362

Eckert, H. M., \& Eichorn, D. H. (1977). Developmental variability in reaction time. Child Development, 48(2), 452-458. doi:10.2307/ 1128638

Enns, J. T., \& Akhtar, N. (1989). A developmental study of filtering in visual attention. Child Development, 60, 1188-1199. doi: $10.2307 / 1130792$
Enns, J. T., \& Girgus, J. S. (1985). Developmental changes in selective and integrative visual attention. Journal of Experimental Child Psychology, 40, 319-337. doi:10.1016/0022-0965(85)90093-1

Forster, S., \& Lavie, N. (2007). High perceptual load makes everybody equal. Psychological Science, 18(5), 377-381. doi:10.1111/j.14679280.2007.01908.x

Frensch, P. A., Lin, J., \& Buchner, A. (1998). Learning versus behavioral expression of the learned: The effects of a secondary tone-counting task on implicit learning in the serial reaction task. Psychological Research, 61, 83-98. doi:10.1007/ s004260050015

Frensch, P. A., Wenke, D., \& Ruenger, D. (1999). A secondary tonecounting task suppresses expression of knowledge in the serial reaction task. Journal of Experimental Psychology. Learning, Memory, and Cognition, 25(1), 260-274. doi:10.1037/02787393.25.1.260

Gilbert, R. (2009). Pure perceptual-based sequence learning: A role for visuospatial attention. Journal of Experimental Psychology. Learning, Memory, and Cognition, 35(2), 528-541. doi:10.1037/ a0014646

Handy, T. C., \& Khoe, W. (2005). Attention and sensory gain control: A peripheral visual process? Journal of Cognitive Neuroscience, 17(12), 1936-1949. doi:10.1162/089892905775008715

Hillyard, S. A., Vogel, E. K., \& Luck, S. J. (1998). Sensory grain control (amplification) as a mechanism of selective attention: Electrophysiological and neuroimaging evidence. Philosophical Transactions of the Royal Society of London. Series B: Biological Sciences, 353(1373), 1257-1270.

Hommel, B., Li, K. Z. H., \& Li, S. (2004). Visual search across the life span. Developmental Psychology, 40(4), 545-558. doi: $10.1037 / 0012-1649.40 .4 .545$

Huang-Pollock, C. L., Carr, T. H., \& Nigg, J. T. (2002). Development of selective attention: Perceptual load influences early versus late attentional selection in children and adults. Developmental Psychology, 38(3), 363-375. doi:10.1037/0012-1649.38.3.363

Jiang, Y., \& Chun, M. M. (2001). Selective attention modulates implicit learning. The Quarterly Journal of Experimental Psychology, 54A (4), 1105-1124. doi:10.1080/02724980042000516

Jiang, Y., \& Leung, A. (2005). Implicit learning of ignored visual context. Psychonomic Bulletin \& Review, 12(1), 100-106.

Klenberg, L., Korkman, M., \& Lahti-Nuuttila, P. (2001). Differential development of attention and executive functions in 3- to 12year-old Finnish children. Developmental Neuropsychology, 20 (1), 407-428. doi:10.1207/S15326942DN2001 6

Korkman, M., Kirk, U., \& Kemp, S. (1998). $\bar{A}$ Developmental Neuropsychological Assessment. San Antonio, TX: The Psychological Corporation

Kunar, M. A., Flusberg, S., Horowitz, T. S., \& Wolfe, J. M. (2007). Does contextual cuing guide the deployment of attention? Journal of Experimental Psychology: Human Perception and Performance, 33(4), 816-828. doi:10.1080/ 13506280701751224

Lavie, N. (1995). Perceptual load as a necessary condition for selective attention. Journal of Experimental Psychology: Human Perception and Performance, 21(3), 451-468. doi:10.1037/00961523.21.3.451

Lavie, N., Hirst, A., de Fockert, J. W., \& Viding, E. (2004). Load theory of selective attention and cognitive control. Journal of Experimental Psychology: General, 133(3), 339-354. doi:10.1037/0096-3445.133.3.339

Mangun, G. R., \& Fannon, S. P. (2007). Attention: Control in the visual cortex. Current Biology, 17(5), R170-R172. doi:10.1016/j. cub.2006.12.028

Mangun, G. R., \& Hillyard, S. A. (1991). Modulations of sensoryevoked brain potentials indicate changes in perceptual processing during visual-spatial priming. Journal of Experimental Psychol- 
ogy: Human Perception and Performance, 17(4), 1057-1074. doi:10.1037/0096-1523.17.4.1057

Mangun, G. R., \& Hillyard, S. A. (1995). Mechanisms and models of selective attention. In M. D. Rugg \& M. G. H. Coles (Eds.), Electrophysiology of Mind: Event-Related Brain Potentials and Cognition (pp. 40-85). New York: Oxford Press.

Maybery, M., Taylor, M., \& O’Brien-Malone, A. (1995). Implicit learning: Sensitive to age but not IQ. Australian Journal of Psychology, 47(1), 8-17. doi:10.1080/00049539508258763

McDermott, J. M., Pérez-Edgar, K., \& Fox, N. (2007). Variations of the flanker paradigm: Assessing selective attention in young children. Behavior Research Methods, 39(1), 62-70.

Meulemans, T., Van der Linden, M., \& Perruchet, P. (1998). Implicit sequence learning in children. Journal of Experimental Child Psychology, 69, 199-221. doi:10.1006/jecp.1998.2442

Newman, R. S., \& Jusczyk, P. W. (1996). The cocktail party effect in infants. Perception \& Psychophysics, 58, 1145-1156.

Nissen, M. J., \& Bullemer, P. (1987). Attentional requirements of learning: Evidence from performance measures. Cognitive Psychology, 19, 1-32. doi:10.1016/0010-0285(87)90002-8

Palmer, J., Verghese, P., \& Pavel, M. (2000). The psychophysics of visual search. Vision Research, 40(10-12), 1227-1268. doi:10.1016/S0042-6989(99)00244-8

Plude, D. J., Enns, J. T., \& Brodeur, D. (1994). The development of selective attention: A life-span overview. Acta Psychologica, 86, 227-272. doi:10.1016/0001-6918(94)90004-3

Ridderinkhoff, R. K., \& van der Stelt, O. (2000). Attention and selection in the growing child: Views derived from developmental psychophysiology. Biological Psychology, 54, 55-106. doi:10.1016/S0301-0511(00)00053-3

Rosas, R., Ceric, F., Tenorio, M., Mourgues, C., Thibaut, C., Hurtado, E., Aravena, M. T. (2010). ADHD children outperform normal children in an artificial grammar implicit learning task: ERP and RT evidence. Consciousness and Cognition: An International Journal, 19(1), 341-351. doi:10.1016/j.con cog.2009.09.006

Rueda, M. R., Fan, J., McCandliss, B. D., Halperin, J. D., Gruber, D. B., Pappert Lercari, L., Posner, M. I. (2004). Development of attention networks in childhood. Neuropsychologia, 42, 1029 1040. doi:10.1016/j.neuropsychologia.2003.12.012

Serences, J. G., Yantis, S., Culberson, A., \& Awh, E. (2004). Preparatory activity in visual cortex indexes distractor suppression during covert spatial orienting. Journal of Neurophysiology, 92, 3538-3545. doi:10.1152/jn.00435.2004

Shanks, D. R., \& Channon, S. (2002). Effects of a secondary task on "implicit" sequence learning: Learning or performance? Psychological Research, 66(2), 99-109. doi:10.1007/s00426-001-0081-2

Slotnick, S. D., Schwarzenbach, J., \& Yantis, S. (2003). Attentional inhibition of visual processing human striate and extrastriate cortex. Neuroimage, 19, 1602-1611. doi:10.1016/S1053-8119(03)00187-3

Stadler, M. A. (1995). Role of attention in implicit learning. Journal of Experimental Psychology. Learning, Memory, and Cognition, 21 (3), 674-685. doi:10.1037/0278-7393.21.3.674

Taylor, M. J., \& Khan, S. C. (2000). Top-down modulation of early selective attention processes in children. International Journal of Psychophysiology, 37, 135-147. doi:10.1016/S0167-8760(00) 00084-2

Thomas, K. M., Hunt, R. H., Vizueta, N., Sommer, T., Durston, S., Yang, Y., Worden, M. S. (2004). Evidence of developmental differences in implicit sequence learning: An fMRI study of children and adults. Journal of Cognitive Neuroscience, 16, 1339-1351. doi:10.1162/0898929042304688

Thomas, K., \& Nelson, C. A. (2001). Serial reaction time learning in preschool and school-age children. Journal of Experimental Child Psychology, 79(4), 364-387. doi:10.1006/jecp.2000.2613

Tipper, S. P., Rafal, R., Reuter-Lorenz, P. A., Starrveldt, Y., Ro, T., Egly, R., ... Weaver, B. (1997). Object-based facilitation and inhibition from visual orienting in the human split-brain. Journal of Experimental Psychology: Human Perception and Performance, 23(5), 1522-1532. doi:10.1037/0096-1523.23.5.1522

Treisman, A., \& Gelade, G. (1980). A feature integration theory of attention. Cognitive Psychology, 12, 97-136. doi:10.1016/00100285(80)90005-5

Vaidya, C. J., Huger, M., Howard, D. V., \& Howard, J. H., Jr. (2007). Developmental differences in implicit learning of spatial context. Neuropsychology, 21(4), 497-506. doi:10.1037/0894-4105.21.4.497 